Relations industrielles

Industrial Relations

\title{
Supiot, Alain, Critique du droit du travail
}

\section{Pierre Verge}

Volume 50, numéro 1, 1995

URI : https://id.erudit.org/iderudit/051005ar

DOI : https://doi.org/10.7202/051005ar

Aller au sommaire du numéro

\section{Éditeur(s)}

Département des relations industrielles de l'Université Laval

\section{ISSN}

0034-379X (imprimé)

1703-8138 (numérique)

Découvrir la revue

Citer ce compte rendu

Verge, P. (1995). Compte rendu de [Supiot, Alain, Critique du droit du travail]. Relations industrielles / Industrial Relations, 50(1), 236-238.

https://doi.org/10.7202/051005ar

Tous droits réservés (C) Département des relations industrielles de l'Université Laval, 1995
Ce document est protégé par la loi sur le droit d'auteur. L’utilisation des services d'Érudit (y compris la reproduction) est assujettie à sa politique d'utilisation que vous pouvez consulter en ligne.

https://apropos.erudit.org/fr/usagers/politique-dutilisation/ 
organisations, cela en marge de la culture dominante. Le présent ouvrage vise justement à contrer ou à corriger cette tendance en s'attaquant à l'étude des phénomènes de sous-cultures à l'intérieur des organisations. L'auteur s'est intéressé plus particulièrement à l'étude des représentations, des significations, des valeurs partagées par les différentes catégories socioprofessionnelles qu'on retrouve dans les grandes organisations. Des catégories, telles que les professionnels spécialisés dans une branche particulière du savoir et des pratiques administratives, des directions supérieures d'entreprises, des cadres intermédiaires, des cadres subalternes, voire même des catégories cols blancs et cols bleus.

La première partie de l'ouvrage met l'accent sur l'étude du phénomène culturel propre à une catëgorie occupationnelle en adaptant une définition de la culture élaborée par des anthropologues et appliquée jadis aux organisations dans leur ensemble. On peut constater que les valeurs, les croyances, le langage diffèrent au passage d'une catégorie occupationnelle à une autre et donnent forme au comportement des membres appartenant à la catégorie. Ceci se traduit extérieurement par l'adoption d'un langage approprié, par des rites de passage distincts et par d'autres mécanismes de socialisation.

La deuxième partie vise à mettre en évidence la dynamique culturelle inhé- rente à l'articulation difficile des relations entre les différentes catégories occupationnelles. À ce sujet, les travaux d'Elton Mayo et de ses collègues avaient déjà signalé la présence de groupes informels au sein des organisations dans la catégorie ouvrière. On se souvient que ces groupes partageaient des visions et des normes différentes de celles de la direction de l'entreprise sous étude, à un point tel qu'on en est venu à voir ces groupes en marge de l'organisation formelle ou en opposition. Dans cette même partie de son ouvrage, l'auteur déborde largement la réalité des groupes informels pour prêsenter une typologie et des modes d'adaptation qui surviennent au sein des interactions entre, par exemple, les souscultures des professionnels et celles des directions générales.

L'auteur en vient ainsi à la fin de son analyse à fairę ressortir les implications de ces différences au plan de la gestion de la diversité culturelle des organisations. Il réussit donc à mettre en évidence que les occupations constituent elles-mêmes des cultures, que l'étude de cette réalité à été fort négligée, due en grande partie à l'engouement qu'on a développé à l'endroit d'une vision plutôt globale de la réalité culturelle des organisations.

\section{LAURENT BÉLANGER} Université Laval

\section{Critique du droit du travail}

par Alain SUPIOT, Paris, P.U.F., 1994, coll. "Les voies du droit ", 280 p., ISBN 2-13-046113-1.

L'ouvrage est d'un apport particulier. Comme l'indique sa prēsentation, "le propos de ce livre n'est pas de décrire le dernier état [d'un droit de travail] en perpétuel changement, mais d'en démonter les ressorts et saisir le principe de son réglage, c'est-à-dire d'en faire la critique au sens philosophique du terme ". Axé sur le développement historique du droit du travail français, il n'hésite pas à sortir de ce champ dès qu'un autre droit national significatif apporte un éclairage essentiel sur le phénomène, qu'il s'agisse, selon le cas, des droits italien, anglais, américain, mais, avant tout, allemand. Il reprend ainsi plusieurs textes antérieurs de l'auteur, du plus récent ("Le travail, liberté partagée", (1993) Droit social 715 , à sa thèse de doctorat, Le juge et le droit du travail, th. 
Bordeaux I, 1979). Une trame, constatet-on ainsi, unissait au fond cette production riche et diversifiée: le sens, la signification du droit du travail dans l'ensemble juridique. De fait, les deux premières parties du livre recherchent le pourquoi de ce droit; la troisième se penche sur ce qu'il est présentement, sa nature scientifique.

L'histoire du droit du travail " a été celle d'une redécouverte progressive de la dimension personnelle [du travail] " (p. 44): le travailleur, sujet de droit, prend le pas sur le travail, bien marchand. Ce droit assure ainsi la sécurité, non seulement physique, mais aussi économique du travailleur; il affirme son identité collective - par exemple par la reconnaissance d'institutions représentatives des travailleurs - aussi bien qu'individuelle: ainsi en est-il notamment du droit à la qualification professionnelle. Cette dynamique correspond en droit français au développement du contenu obligatoire du contrat de travail contemporain, par opposition à la conception réductrice du louage de services. Un chapitre préliminaire avait toutefois bien souligné la relativité de l'instrument contractuel utilisé en la comparant à la tradition statutaire germaniste. Un statut, fruit aussi bien de l'action étatique que de l'autonomie collective, s'est donc graduellement inséré dans le contrat en droit français. L'auteur répond ainsi à sa première question: "Le travail qui met la personne en rapport avec les choses, est-il chose ou personne? ". Et de poser résolument que ce contrat de travail, même s'il n'engage pas la personne toute entière, "a le corps pour objet ". Le contrat de travail se distingue ainsi du contrat d'entreprise et Alain Supiot lui-même, de la thèse récente de Thierry Revet, pour qui l'objet du contrat de travail est la force de travail du salarié (La force de travail, Th. Montpellier, 1991, Paris, Litec, 1992, p. 59). Encore plus à fond (Partie II), le droit du travail doit essentiellement concilier subordination et liberté du tra- vailleur. Deuxième question de l'auteur, qui animait déjà sa thèse de doctorat : "Un homme libre peut-il être soumis au pouvoir de son égal?". Telle soumission ne peut procéder que de l'autono mie contractuelle; au droit du travail d'organiser cette soumission! Son rôle essentiel est, à la fois, de légaliser et de limiter le pouvoir patronal, de civiliser l'entreprise, d'y définir des aires d'autonomie collective et individuelle.

La troisième partie, de nature épistémologique ("Le légal et le normal "), affirme d'emblée la place de ce droit issu de l'appréhension sociologique du travail subordonné dans l'univers juridique: il est bien rationalité, mais cette rationalité, si on la compare au construit systématique du droit civil, est davantage matérielle que formelle. Place à la critique de la légalisation. Celle de l'heure se fonde davantage sur Hayek que sur Marx: ce droit du travail ne serait-il qu'une somme superflue, voire nocive de "règles d'organisations * ? La réponse est plurale: les accords collectifs, les grèves et les syndicats ont d'abord existé dans les faits avant d'être légalisés; supprimons le droit du travail et le pouvoir d'organisation du chef d'entreprise comble absolument le vide; le droit du travail véhicule dans l'entreprise des principes essentiels de la société libre et il est fait pour une bonne part de règles générales d'encadrement. La règle de droit doit cependant conserver son essence, se distinguer de la norme domestique, qui n'est que pouvoir de gouvernement. Il y a aussi le danger de l'envahissement - l'expérience communautaire illustre - de la norme technique, celui de la normalisation des "ressources humaines ": au juriste, vœu ultime (p. 268), de "rappeler la raison gestionnaire à l'ordre du sujet de droit, lorsqu'elle se laisse égarer par la magie des chiffres "!

Mais, le travail lui-mēme se transforme profondément : diversification, individualisation des statuts, dualisation du marché du travail... Il devient même un 
bien rare. La conclusion s'ouvre sur ces mutations et incite à une réflexion renouvelée sur le sens que doit maintenant revêtir la liberté du travail (p. 257). Un seul regret du lecteur, compte tenu de l'ampleur de la réflexion de l'auteur sur le travail et le droit: le caractère relativement succinct du passage consacré, dans ce contexte mouvant, au droit du travail. (Quid, par exemple, les vues de A. Gorz, Métamorphoses du travail - Quête de sens, Paris, Galilée, 1988 ?)

D'une façon toute particulière, le lecteur nord-américain pourra se sensibiliser encore davantage au particularisme, à certains égards, du droit du travail français, même si l'ouvrage s'inscrit plus généralement dans l'histoire universelle de cette branche du droit. À la différence d'autres traditions, l'autonomie collective - liberté syndicale, droit de grève, droit à la négociation collective - n'y est pas conçue primordialement en termes "d'organisation communautaire". Elle s'y affirme en tant que liberté publique, au profit de tout salarié. Il en résulte un ordre public social, statut qui loge dans chaque contrat individuel de travail (p. 137). D'où, par exemple, la possibilité pour celui-ci de déroger in mejus à la règle collective (p. 140).

"Petit livre", dixit modestement l'auteur, dont le contenu n'a pu être ici que fort imparfaitement évoqué - chaque page est nourrie d'une réflexion et d'une culture impressionnantes -, mais livre plutôt unique de réflexion essentielle et universelle sur le droit du travail, dans la mesure du moins où il est possible de l'abstraire de la diversité des droits nationaux.

PIERRE VERGE

Université Laval

\section{Le droit disciplinaire des corporations professionnelles}

par Mario Goulet, Cowansville, Éditions Yvon Blais, 1993, 238 p., ISBN 2-89073-888-4.

Compte tenu des critiques fréquentes formulées à l'endroit des corporations professionnelles, l'intérêt d'une étude de l'action disciplinaire au sein de ces organismes publics apparait évident. De plus, qui n'a pas d'idées préconçues au sujet des devoirs et de la responsabilité des professionnels? En raison même de ces préjugés à l'égard des professionnels, ce champ d'étude comporte d'importants et délicats obstacles. Aussi, estce tout à l'honneur de $\mathrm{M}^{\mathrm{e}}$ Mario Goulet d'avoir entrepris pareille aventure.

La faute, élément essentiel, doit d'abord être établie et cette opération implique que l'on circonscrive convenablement les devoirs et obligations des professionnels. Cette opération s'effectue d'abord d'une façon objective puis, en tenant compte de certaines circonstances particulières de chaque affaire. La faute, et ses effets à l'égard des profes- sionnels, des collègues et du public ne peuvent être des données faciles à saisir et à jauger. Ce sont néanmoins les deux pôles de cette courte étude de $M^{e}$ Goulet rédigée initialement en vue de l'obtention d'une maitrise ( $2^{\mathrm{e}}$ cycle) en droit.

La deuxième moitié des deux cent trente-huit pages de l'ouvrage est constituée d'annexes: le Code des professions, une bibliographie, l'index des décisions rapportées, etc. La première partie de cette monographie comprend un grand nombre de citations et de renvois infrapaginales (plus de quatre cents). À ce seul point de vue, il s'agit d'une éclatante démonstration de l'observation de Pascal à l'effet que dans tout texte, il y a souvent plus de bien des autres que du sien. Pareille facture présentée pour les fins d'un deuxième cycle d'études universitaires demeure 